\title{
Ultrafast Quenching of the Exchange Interaction in a Mott Insulator
}

\author{
J. H. Mentink* and M. Eckstein \\ Max Planck Research Department for Structural Dynamics, University of Hamburg-CFEL, 22761 Hamburg, Germany
}

(Received 20 January 2014; published 29 July 2014)

\begin{abstract}
We investigate how fast and how effective photocarrier excitation can modify the exchange interaction $J_{\text {ex }}$ in the prototype Mott-Hubbard insulator. We demonstrate an ultrafast quenching of $J_{\text {ex }}$ both by evaluating exchange integrals from a time-dependent response formalism and by explicitly simulating laser-induced spin precession in an antiferromagnet that is canted by an external magnetic field. In both cases, the electron dynamics is obtained from nonequilibrium dynamical mean-field theory. We find that the modified $J_{\text {ex }}$ emerges already within a few electron hopping times after the pulse, with a reduction that is comparable to the effect of chemical doping.
\end{abstract}

DOI: 10.1103/PhysRevLett.113.057201

PACS numbers: 75.78.Jp, 71.10.Fd

Magnetic long-range order and the dynamics of spins in magnetic materials are governed by the exchange interaction $J_{\mathrm{ex}}$, the strongest force of magnetism. Because $J_{\mathrm{ex}}$ emerges from the Pauli principle and the electrostatic Coulomb repulsion between electrons, it is sensitive to purely nonmagnetic perturbations. This fact implies intriguing and largely unexplored possibilities for the ultrafast control of magnetism by femtosecond laser pulses, which is currently a very active research area [1]. In principle, laser excitation can effect $J_{\text {ex }}$ by modulating the electronic structure (electron hopping, Coulomb repulsion) and by creating a nonequilibrium distribution of photoexcited carriers (photodoping). A modification of $J_{\text {ex }}$ has been discussed within the context of experiments on manganites [2-4], magnetic semiconductors [5], and, using static field gradients, ultracold atoms in optical lattices $[6,7]$. While it might play a role as well in metallic ferromagnets [8-11], ultrafast demagnetization [12] and laser-induced magnetization reversal [13-15] seem at least partly understood in terms of a given time-independent $J_{\mathrm{ex}}$. Clearly, more theoretical work is needed to understand how effective a modification of $J_{\text {ex }}$ under nonequilibrium conditions can be and how fast $J_{\text {ex }}$ can be modified. The latter touches the fundamental question for the time scale at which the description of spin dynamics in terms of a $J_{\text {ex }}$ emerges from the full electronic dynamics, before which $J_{\text {ex }}$ is not a valid concept at all. Although this question has not been directly addressed in the experiments mentioned above, an investigation of this ultimate limit of spin dynamics is in range using today's femtosecond laser technology.

In general, the exchange interaction arises from a lowenergy description of the electronic states in terms of magnetic degrees of freedom. Recently, Secchi et al. defined the nonequilibrium exchange interaction via an effective action that governs the spin dynamics out of equilibrium, leading to an expression in terms of nonequilibrium electronic Green's functions [16]. Here, we apply this framework to the paradigm single-band MottHubbard insulator at half-filling, for which the concept of exchange interaction in equilibrium is very well understood. To directly assess the nonequilibrium electron dynamics and evaluate the nonequilibrium Green's functions, we employ nonequilibrium dynamical mean field theory (DMFT). Previous investigations of the antiferromagnetic phase in the Hubbard model have demonstrated ultrafast melting of long-range order after an interaction quench $[17,18]$. Here, we will focus on the excitation with an electric field pulse and weaker excitation strength, to assess the control of $J_{\text {ex }}$ within the ordered phase and to determine how fast a rigid spin dynamics emerges after the excitation.

Model.-In this Letter, we study the antiferromagnetic phase of the repulsive Hubbard model at half-filling,

$$
H=-t_{0} \sum_{\langle i j\rangle \sigma} c_{i \sigma}^{\dagger} c_{j \sigma}+U \sum_{j} n_{j \uparrow} n_{j \downarrow}+B_{x} \sum_{j} S_{j x} .
$$

Here, $c_{i \sigma}^{\dagger}$ creates an electron at site $i$ with spin $\sigma=\uparrow, \downarrow$ along a given spin quantization axis (the $z$ axis). The first two terms describe nearest-neighbor hopping $t_{0}$ and repulsive on-site interaction $U$. The third term introduces coupling of the spin $S_{j \alpha}=\frac{1}{2} \sum_{\sigma \sigma^{\prime}} c_{j \sigma}^{\dagger}\left(\hat{\sigma}_{\alpha}\right)_{\sigma \sigma^{\prime}} c_{j \sigma^{\prime}}$ to a homogeneous magnetic field $B_{x}$ along the $x$ axis $\left(\alpha=x, y, z ; \hat{\sigma}_{\alpha}\right.$ denote the Pauli matrices). The latter allows us to probe transverse dynamics of the antiferromagnetic order parameter in the $y-z$ plane; the $x$ component of the total spin $\left\langle S_{x}\right\rangle$ is conserved.

To solve the electron dynamics in the Hubbard model, we use nonequilibrium DMFT [19,20]. Within DMFT [21], which becomes exact in the limit of infinite dimensions [22], local correlation functions are obtained from an effective impurity model in which one site of the lattice is coupled to a noninteracting bath. In the presence of a transverse magnetic field $B_{x}$ one must include spin-flip terms in the effective impurity action, which thus takes the 
form $\mathcal{S}=\mathcal{S}_{\mathrm{loc}}-i \int d t \int d t^{\prime} \sum_{\sigma \sigma^{\prime}} c_{\sigma}(t)^{\dagger} \Delta_{\sigma \sigma^{\prime}}\left(t, t^{\prime}\right) c_{\sigma^{\prime}}\left(t^{\prime}\right)$. Here, $\Delta_{\sigma \sigma^{\prime}}\left(t, t^{\prime}\right)$ is the hybridization function of the bath that is determined self-consistently. The impurity model is solved within the perturbative hybridization expansion (noncrossing approximation, NCA). The incorporation of spin-flip terms $\Delta_{\uparrow \downarrow}$ is a straightforward extension to the nonequilibrium DMFT implementation and the NCA, which have been explained in Refs. [20] and [23]. For completeness, we summarize explicit equations in the Supplemental Material [24]. In general, the DMFT approximation is expected to be appropriate when local correlations dominate, such as is the case in the Mott-insulating phase for the short-time dynamics (up to $\sim 100 \mathrm{fs}$ ), when the much slower ( $\sim$ ps and beyond) inhomogeneous dynamics (spin waves, domain growth) is not yet developed. The reliability of the NCA impurity solver has been tested in equilibrium and for short-time dynamics by comparison with higher-order hybridization expansions as well as with numerically exact quantum Monte Carlo impurity solver. Good agreement was found at large $U$ in the paramagnetic phase $[23,25]$ and for the antiferromagnetic phase boundary [17].

Nonequilibrium exchange interactions.-For general nonequilibrium situations, the exchange interaction is defined in terms of an effective spin action that reproduces the spin dynamics of the full electronic model. A formal derivation of the spin interaction in such a model has been given by Secchi and co-workers [16]. The essential idea is to define the effective spin action in terms of timedependent rotations of the spin quantization axes $\mathbf{e}_{i}(t)$, as described by Holstein-Primakov bosons $\xi_{i}(t)$. Starting from the electronic partition function as a path integral over fermionic fields $\phi$, one introduces rotated fermion fields $\psi$ and then expands the action to second order in $\xi$. The rotated fermionic fields are integrated out, which leads to spin action with an interaction term of the form $\mathcal{S}_{\text {spin }}\left[\xi^{*}, \xi\right]=\sum_{i j} \int d t \int d t^{\prime} \xi_{i}^{*}(t) A_{i j}\left(t, t^{\prime}\right) \xi_{j}\left(t^{\prime}\right)$. The coupling $A_{i j}\left(t, t^{\prime}\right)$ between spin rotations at different times and different sites $i \neq j$ is expressed in terms of the spindependent single-particle Green's functions $G_{i j}^{\sigma}\left(t, t^{\prime}\right)$ and the self-energies $\Sigma_{i j}^{\sigma}\left(t, t^{\prime}\right)$,

$$
\begin{aligned}
A_{i j}\left(t, t^{\prime}\right)= & R_{i j}^{\downarrow}\left(t, t^{\prime}\right) R_{j i}^{\uparrow}\left(t^{\prime}, t\right)+S_{i j}^{\downarrow}\left(t, t^{\prime}\right) S_{j i}^{\uparrow}\left(t^{\prime}, t\right) \\
& -T_{i j}^{\downarrow}\left(t, t^{\prime}\right) G_{j i}^{\uparrow}\left(t^{\prime}, t\right)-G_{i j}^{\downarrow}\left(t^{\prime}, t\right) T_{j i}^{\uparrow}\left(t^{\prime}, t\right),
\end{aligned}
$$

where $T_{i j}^{\sigma}\left(t, t^{\prime}\right)=\Sigma_{i j}^{\sigma}\left(t, t^{\prime}\right)+[\Sigma \cdot G \cdot \Sigma]_{i j}^{\sigma}\left(t, t^{\prime}\right), R_{i j}^{\sigma}\left(t, t^{\prime}\right)=$ $[G \cdot \Sigma]_{i j}^{\sigma}\left(t, t^{\prime}\right)$, and $S_{i j}^{\sigma}\left(t, t^{\prime}\right)=[\Sigma \cdot G]_{i j}^{\sigma}\left(t, t^{\prime}\right)$. These formulas are a direct generalization of the equilibrium formalism $[26,27]$, which is based on variations of the total (free) energy $\delta E=J_{\text {ex }} \theta^{2}$ for static spin rotations by a small angle $\theta$. We emphasize that Eq. (2) is valid for arbitrary fast and strong fields, apart from neglecting of vertex corrections [16]. In addition, the expressions assume rotations from a collinear state. Reduction of the action with a retarded (two-time) exchange coupling to a spin Hamiltonian with an instantaneous (possibly time-dependent) interaction is possible when the rotations of the quantization axes are much slower than the electron dynamics and, in particular, slower than time-dependent fluctuations of the local magnetic moments themselves. Then, we can average over the fast electron dynamics,

$$
J_{i j}(t)=\operatorname{Im} \int_{0}^{\infty} d s A_{i j}^{\mathrm{ret}}(t, t-s) .
$$

Still, $J_{i j}(t)$ contains not only the exchange interactions but also the time-averaged reduction of the local spin by fluctuations. The "bare" exchange interactions between spin vectors $\left\langle\mathbf{S}_{i}\right\rangle$ are finally given by

$$
J_{i j}^{0}(t)=\frac{1}{4} \frac{J_{i j}(t)}{\left\langle S_{i z}\right\rangle\left\langle S_{j z}\right\rangle} .
$$

In the regime where a rigid macrospin model is valid, $J_{i j}^{0}$ should determine the spin dynamics by a Landau-Lifshitz equation. For a canted antiferromagnet on a bipartite lattice in a transverse magnetic field $B_{x}$, we can write $\left\langle\dot{\mathbf{S}}_{1}\right\rangle=$ $-\left\langle\mathbf{S}_{1}\right\rangle \times \mathbf{B}_{\text {eff }}$, where $\mathbf{B}_{\text {eff }}=2 J_{\text {ex }}\left\langle\mathbf{S}_{2}\right\rangle+B_{x} \mathbf{e}_{x}$. Here, $\left\langle\mathbf{S}_{1,2}\right\rangle$ correspond to the spin on the two sublattices, and the effective exchange interaction is $J_{\mathrm{ex}}=\sum_{j} J_{i j}^{0}$. Using Néel symmetry $\left\langle S_{1 y, z}\right\rangle=-\left\langle S_{2 y, z}\right\rangle,\left\langle S_{1 x}\right\rangle=+\left\langle S_{2 x}\right\rangle$ we can infer the exchange interaction in the canted geometry from the spin dynamics,

$$
J_{\mathrm{ex}}^{c}=-\frac{B_{x}}{4\left\langle S_{1 x}\right\rangle}-\frac{1}{4\left\langle S_{1 x}\right\rangle} \frac{\left\langle\dot{S}_{1 y}\right\rangle}{\left\langle S_{1 z}\right\rangle} .
$$

The validity of the instantaneous approximation is a fundamental question that is not resolved in general and that will be partially addressed below by comparison of the two Eqs. (4) and (5).

Results.-We first solve the DMFT equations on the Bethe lattice with a semielliptic density of states $D(\epsilon)=\sqrt{4-\epsilon^{2}} / 2 \pi$. This setup implies a closed-form self-consistency condition and allows us to compute the electronic dynamics to long times, as needed for an accurate evaluation of the integral in Eq. (3) (see the Supplemental Material [24]).

Before exploring nonequilibrium, it is illustrative to evaluate the exchange interaction (4) in the familiar equilibrium case. For the Mott insulator at half-filling, the static exchange interaction at zero temperature can be obtained from a perturbation expansion in the hopping, which yields $\left|J_{\mathrm{ex}}^{a}\right|=2 t_{0}^{2} / U$. In Fig. 1, we compare the analytical value $\left|J_{\mathrm{ex}}^{a}\right|$ (dashed lines) and the bare exchange interaction $\left|J_{\text {ex }}^{0}\right|=\left|J_{12}^{0}\right|$ computed from the collinear DMFT solution using Eq. (4) (red circles) as a function of temperature for three values of $U$. In addition, we solve the DMFT equations for the antiferromagnetic Mott 


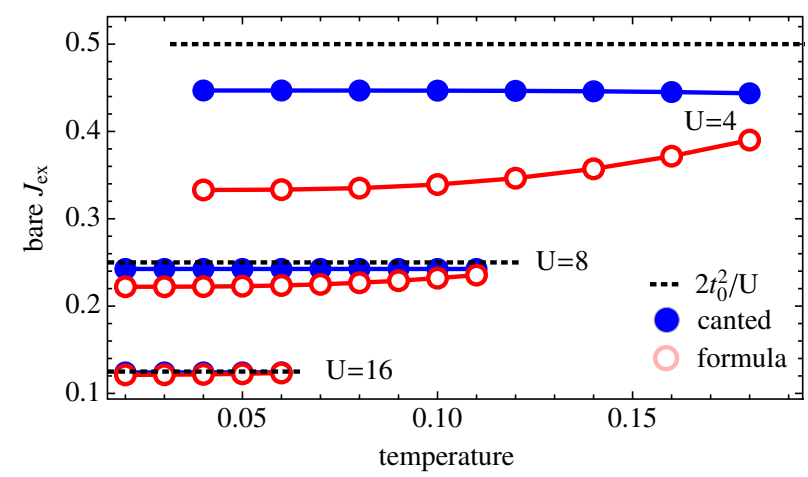

FIG. 1 (color online). Bare exchange interaction as function of temperature for different values $U$, computed from the formula Eq. (4) (red open circles) and from the canted geometry Eq. (5) (blue solid disks). For large $U$ the calculations show excellent agreement with the analytical result $\left|J_{\mathrm{ex}}^{a}\right|=2 t_{0}^{2} / U$ indicated with dashed lines.

insulator in a weak transverse field of strength $B_{x}$ and obtain an estimate $\left|J_{\mathrm{ex}}^{c}\right|=\left|B_{x} / 4\left\langle S_{x}\right\rangle\right|$ by comparing the canting $\left\langle S_{x}\right\rangle$ of spins to the prediction from a rigid macrospin model, Eq. (5), in the static limit (blue solid disks). We choose $B_{x}=0.64 t_{0}^{2} / U$, such that the canting angle at low temperature is about 10 degrees for all $U$. At large $U$, we find excellent agreement between $J_{\mathrm{ex}}^{a}, J_{\mathrm{ex}}^{0}$, and $J_{\mathrm{ex}}^{c}$, where deviations between $J_{\mathrm{ex}}^{c}$ and $J_{\mathrm{ex}}^{a}$ are on the order of $\left(t_{0} / U\right)^{2}$, which also confirms the validity of the DMFT approximation for studying exchange interactions. For smaller $U$, the deviation of $J_{\mathrm{ex}}^{0}$ from $J_{\mathrm{ex}}^{c}$ becomes more pronounced, up to $25 \%$ at $U=4$. The differences between the two expressions of Eqs. (4) and (5) may have several possible origins: (i) At small values of $U$, the rigid macrospin model is no longer valid, because retardation effects in $A\left(t, t^{\prime}\right)$ become relevant, (ii) vertex corrections to Eq. (2) become important, or (iii) $J_{\mathrm{ex}}^{0}$ is a nearest-neighbor interaction while Eq. (5) also takes into account nextnearest-neighbor terms. Below, we will study nonequilibrium exchange at large values of $U$. Nevertheless, for moderate $U$, where retardation effects to the exchange become important, we can still use Eq. (5) as a heuristic measure for $J_{\mathrm{ex}}$, in the sense that it is the best estimate of an instantaneous exchange interaction which is in accordance with an observed spin dynamics.

Next, we investigate how fast $J_{\text {ex }}$ can be modified under electronic nonequilibrium conditions, which we generate by suddenly changing $U$. It was recently demonstrated that after such an interaction quench the order parameter $m$ quickly relaxes to a quasistationary but nonthermal value [17] that is protected from further decay by the slow recombination rate of doublons and holes [28-31]. This transient state resembles properties of a photodoped system in which charge carriers are created by a short laser pulse. We will refer to the induced change of the doublon and hole densities $d$ and $h$ with respect to their

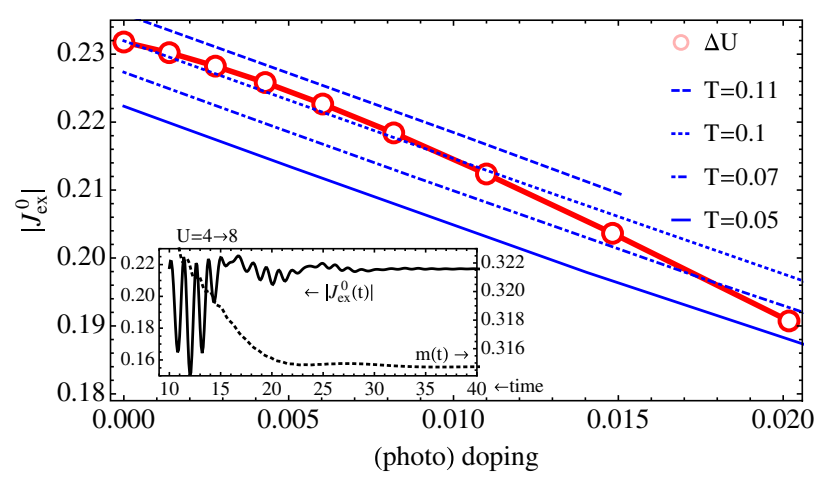

FIG. 2 (color online). Comparison of the nonequilibrium exchange interaction (open circles) in the quasistationary state after an interaction quench $\Delta U$ in the Bethe lattice with the equilibrium exchange interaction of the chemically doped model (solid symbols) for $U=8$ and different temperatures. The inset shows the bare time-dependent exchange interaction (solid line) and staggered magnetization (dashed line) caused by the quench $U=4 \rightarrow 8$.

equilibrium values $d_{0}$ and $h_{0}$ as photodoping $\Delta n=d+h-d_{0}-h_{0}=2\left(d-d_{0}\right)$. The inset of Fig. 2 shows the evolution of the time-dependent nonequilibrium exchange interaction (solid line) and order parameter (dashed line) for a quench $U=4 \rightarrow 8$. [A Gaussian window $\exp \left(-s^{2} / w^{2}\right)$ of length $w=10 t_{0} / \pi$ was used in Eq. (3) to ensure a smooth cutoff of the upper integration limit.] We find that $\left|J_{\mathrm{ex}}^{0}\right|$, like $m$, becomes stationary already on an electronic time scale, which shows the emergence of a spin Hamiltonian on the time scale of a few tens of inverse hoppings.

To study how effective $J_{\mathrm{ex}}$ is modified, we evaluate it in the quasistationary state after different excitation strengths $\Delta U=U_{f}-U_{i}=0, \ldots, 4$, with final $U_{f}=8$. The result is shown by red open circles in Fig. 2 as a function of "photodoping" $\Delta n$, demonstrating a reduction of $J_{\text {ex }}$ to a value significantly below the equilibrium difference $J_{\mathrm{ex}}^{0}\left(U_{i}\right)-$ $J_{\mathrm{ex}}^{0}\left(U_{f}\right)$. The results are independent of a Gaussian cutoff in Eq. (3) for $w=60 t_{0} / \pi$. Only for the largest $\Delta U$ do we find a slight dependence on $w$ that indicates that $J_{\text {ex }}^{0}$ is not yet fully stationary. Furthermore, the blue lines in Fig. 2 show the equilibrium exchange interaction at chemical doping for different temperatures. These results confirm the conclusions obtained from analyzing the electronic spectrum [17], that properties of the photodoped state with added doublons and holes resemble those of the chemically doped state with the same total number of carriers: Adding doublons and holes causes an ultrafast weakening, or "quenching" of the exchange interaction by an amount comparable to that of chemical doping. Qualitatively, the weakening of the antiferromagnetic exchange can result from a lowering of the kinetic energy of mobile carriers in a parallel spin alignment (for $U=\infty$ and small doping ferromagnetism is favored [32]). 


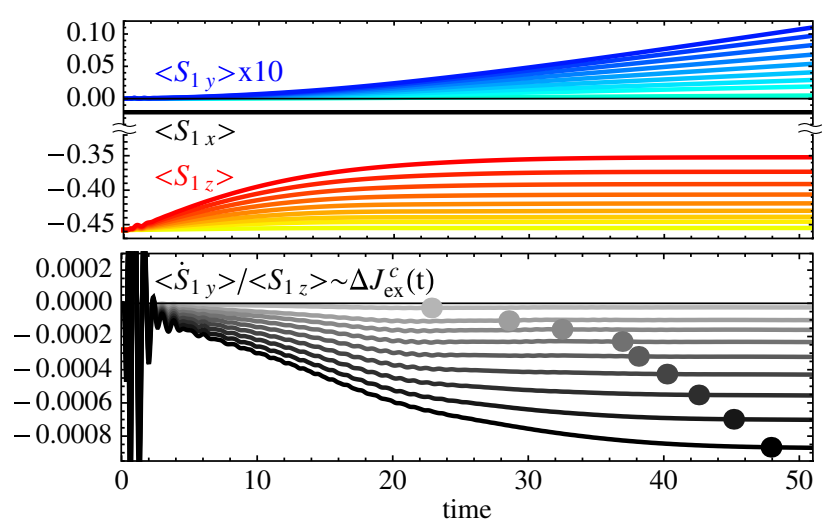

FIG. 3 (color online). Induced spin dynamics (top) and modification of the exchange interaction (bottom) caused by excitation with an electric field (hypercubic lattice, $U=8$ ).

Photoexcitation.-To further demonstrate the possibility of changing $J_{\mathrm{ex}}$ in a setup that is closer to the laser excitation of condensed-matter systems, we study the Hubbard model driven by an external electric field. This is implemented for the infinite-dimensional hypercubic lattice with density of states $D(\epsilon)=\exp \left(-\epsilon^{2}\right) / \sqrt{\pi}$, with the electric field pointing along the body diagonal $[20,33]$. Photoexcited carriers are created by a single-cycle pulse $E(t)=E_{0} \sin (\omega t) \exp \left[-\alpha\left(t-t_{c}\right)^{2}\right], t_{c}=\pi / \omega, \alpha=4.6 / t_{c}^{2}$ with a Gaussian envelope and a center frequency of $\omega=U$. To directly measure the transverse spin dynamics associated with $J_{\text {ex }}$, we study the system in a canted geometry induced by a homogeneous magnetic field $B_{x}$. Before laser excitation, the system is prepared in equilibrium with a canting angle determined by the balance of $B_{x}$ and $J_{\mathrm{ex}}$. When $J_{\text {ex }}$ is changed, this balance will be broken and a spin resonance will be excited. Such spin resonances can, in principle, be detected experimentally using magnetooptical techniques [1] and $\mathrm{THz}$ spectroscopy [34]. In our simulations, we extract the nonequilibrium exchange interaction by comparing the spin dynamics obtained within DMFT to the rigid macrospin model; cf. Eq. (5). The results of this approach are shown in Fig. 3, computed at $U=8$, $B_{x}=0.01$, and initial temperature $T=0.03$. The top panel shows that the sublattice magnetization is initially in the $x-z$ plane. Light to dark colors indicate excitation strengths ranging from $\left|E_{0}\right| / t_{0}=1$ to 5.5 . The bottom panel shows $\Delta J_{\mathrm{ex}}^{0} \sim\left\langle\dot{S}_{1 y}\right\rangle /\left\langle S_{1 z}\right\rangle$ [cf. Eq. (5)], where $\left\langle\dot{S}_{1 y}\right\rangle$ is computed from the time trace of $\left\langle S_{1 y}(t)\right\rangle$. We observe three different time scales in our simulations: (i) Fast $1 / U$ oscillations on the time scale of the laser excitation, as most clearly seen in the bottom panel. This characterizes the stabilization of the local magnetic moments. (ii) Relaxation of the order parameter and the exchange interaction. (iii) The onset of rigid rotation of the spin sublattices at quasistationary values $\left|\left\langle\mathbf{S}_{1}\right\rangle\right|$ and $J_{\mathrm{ex}}^{c}$. We estimate the time $t_{*}$ that it takes for $J_{\mathrm{ex}}^{c}$ to become stationary from $J_{\mathrm{ex}}^{c}\left(t_{*}\right)-J_{\mathrm{ex}}^{c}\left(t_{\mathrm{max}}\right)<\varepsilon$, where $\varepsilon$ is the numerical accuracy. The values $t_{*}$, which are

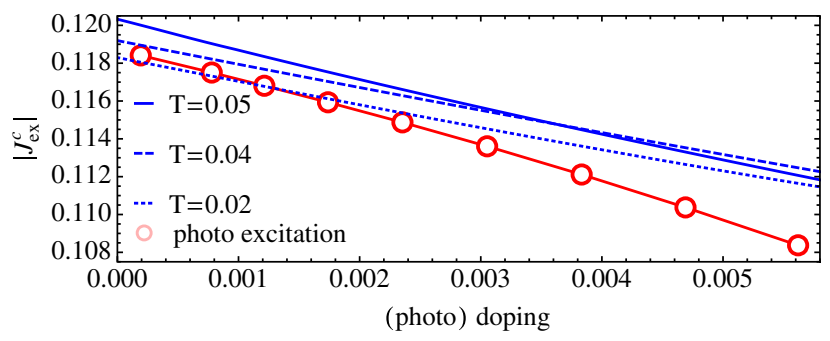

FIG. 4 (color online). Comparison of the nonequilibrium exchange interaction (red open circles) computed from the induced precession (Fig. 3), with the equilibrium exchange interaction in the chemically doped system (blue lines).

indicated as dots in the bottom panel of Fig. 3, show that a quasistationary state and rigid spin dynamics emerge after a few tens of hoppings, similar as for the sudden change of $U$. This relaxation time increases with the excitation density, as the critical excitation for melting the antiferromagnetic order is approached, but it is much shorter than the period of spin precession in the field, which supports the interpretation that photoexcitation causes an ultrafast quenching of $J_{\mathrm{ex}}$. Furthermore, we find that direct photoexcitation has a similar effect as the interaction quench; i.e., the efficiency of the modification of $J_{\mathrm{ex}}$ is determined by the number of photoexcited carriers. This is demonstrated in Fig. 4 by plotting the extracted exchange interaction in the quasistationary state as a function of the photodoping, together with equilibrium calculations in the canted geometry with chemical doping. In the hypercubic lattice, we observe that photoexcitation modifies $J_{\mathrm{ex}}^{c}$ slightly stronger than chemical doping. In addition, there is a more pronounced temperature dependence of $J_{\mathrm{ex}}^{c}$ in equilibrium. Both effects might be related to a slightly different dynamics of low-energy (photo-) doped carriers in the Bethe lattice and the hypercubic lattice, where the latter does not have a sharp band edge in the density of states.

In summary, we report that photoexcitation causes an ultrafast quenching of the exchange interaction in a Mott insulator. An effectively static $J_{\text {ex }}$ can be defined already on the ultrafast time scale on the order of a few tens of inverse hopping times, which is similar to the relaxation time of the order parameter. The reduction of $J_{\mathrm{ex}}$ is comparable to that of a chemically doped state when measured in terms of the total number of excited carriers. These results demonstrate intriguing possibilities to control magnetic order without magnetic fields. Similar or even more efficient ways to control $J_{\text {ex }}$ under nonequilibrium conditions might be found by extending our work to more complex multi-band systems such as the prototype Mott-insulator $\mathrm{V}_{2} \mathrm{O}_{3}$ [35] and to materials with different exchange mechanisms.

We thank K. Balzer, S. Brener, A. Secchi, M. I. Katsnelson, A. V. Kimel, J. Kroha, A. Lichtenstein, and $\mathrm{Ph}$. Werner for fruitful discussions. The calculations were 
run on the supercomputer HLRN-II of the North-German Supercomputing Alliance. J. H. M. acknowledges funding from the Nederlandse Organisatie voor Wetenschappelijk Onderzoek (NWO Rubicon-grant).

*Johan.Mentink@mpsd.cfel.de

[1] A. Kirilyuk, A. V. Kimel, and T. Rasing, Rev. Mod. Phys. 82, 2731 (2010).

[2] S. Wall, D. Prabhakaran, A. T. Boothroyd, and A. Cavalleri, Phys. Rev. Lett. 103, 097402 (2009).

[3] M. Först, R. I. Tobey, S. Wall, H. Bromberger, V. Khanna, A. L. Cavalieri, Y.-D. Chuang, W. S. Lee, R. Moore, W. F. Schlotter, J. J. Turner, O. Krupin, M. Trigo, H. Zheng, J. F. Mitchell, S. S. Dhesi, J. P. Hill, and A. Cavalleri, Phys. Rev. B 84, 241104 (2011).

[4] T. Li, A. Patz, L. Mouchliadis, J. Yan, T. A. Lograsso, I. Perakis, and J. Wang, Nature (London) 496, 69 (2013).

[5] M. Matsubara, A. Schroer, A. Schmehl, A. Melville, C. Becher, M. Martinez, D. Schlom, J. Mannhart, J. Kroha, and M. Fiebig, arXiv:1304.2509.

[6] L.-M. Duan, E. Demler, and M. D. Lukin, Phys. Rev. Lett. 91, 090402 (2003).

[7] S. Trotzky, P. Cheinet, S. Flling, M. Feld, U. Schnorrberger, A. M. Rey, A. Polkovnikov, E. A. Demler, M. D. Lukin, and I. Bloch, Science 319, 295 (2008).

[8] G. Ju, J. Hohlfeld, B. Bergman, R. J. M. van de Veerdonk, O. N. Mryasov, J.-Y. Kim, X. Wu, D. Weller, and B. Koopmans, Phys. Rev. Lett. 93, 197403 (2004).

[9] J. Thiele, M. Buess, and C. H. Back, Appl. Phys. Lett. 85, 2857 (2004).

[10] H.-S. Rhie, H. A. Dürr, and W. Eberhardt, Phys. Rev. Lett. 90, 247201 (2003).

[11] R. Carley, K. Döbrich, B. Frietsch, C. Gahl, M. Teichmann, O. Schwarzkopf, P. Wernet, and M. Weinelt, Phys. Rev. Lett. 109, 057401 (2012).

[12] E. Beaurepaire, J.-C. Merle, A. Daunois and J.-Y. Bigot, Phys. Rev. Lett. 76, 4250 (1996).

[13] C. D. Stanciu, F. Hansteen, A. V. Kimel, A. Kirilyuk, A. Tsukamoto, A. Itoh, and T. Rasing, Phys. Rev. Lett. 99, 047601 (2007).

[14] I. Radu, K. Vahaplar, C. Stamm, T. Kachel, N. Pontius, H. A. Dürr, T. A. Ostler, J. Barker, R. F. L. Evans, R. W. Chantrell, A. Tsukamoto, A. Itoh, A. Kirilyuk, T. Rasing, and A. V. Kimel, Nature (London) 472, 205 (2011).

[15] T. Ostler, J. Barker, R. Evans, R. Chantrell, U. Atxitia, O. Chubykalo-Fesenko, S. El Moussaoui, L. Le Guyader, E.
Mengotti, L. Heyderman, F. Nolting, A. Tsukamoto, A. Itoh, D. Afanasiev, B. Ivanov, A. Kalashnikova, K. Vahaplar, J. Mentink, A. Kirilyuk, T. Rasing, and A. Kimel, Nat. Commun. 3, 666 (2012).

[16] A. Secchi, S. Brener, A. Lichtenstein, and M. Katsnelson, Ann. Phys. (Amsterdam) 333, 221 (2013).

[17] P. Werner, N. Tsuji, and M. Eckstein, Phys. Rev. B 86, 205101 (2012).

[18] N. Tsuji, M. Eckstein, and P. Werner, Phys. Rev. Lett. 110, 136404 (2013).

[19] J. K. Freericks, V. M. Turkowski, and V. Zlatić, Phys. Rev. Lett. 97, 266408 (2006).

[20] H. Aoki, N. Tsuji, M. Eckstein, M. Kollar, T. Oka, and P. Werner, Rev. Mod. Phys. 86, 779 (2014).

[21] A. Georges, G. Kotliar, W. Krauth, and M. J. Rozenberg, Rev. Mod. Phys. 68, 13 (1996).

[22] W. Metzner and D. Vollhardt, Phys. Rev. Lett. 62, 324 (1989).

[23] M. Eckstein and P. Werner, Phys. Rev. B 82, 115115 (2010).

[24] See the Supplemental Material at http://link.aps.org/ supplemental/10.1103/PhysRevLett.113.057201 for methodical aspects of the implementation of nonequilibrium DMFT with a transverse field and the evaluation of the exchange integrals.

[25] M. Eckstein and P. Werner, Phys. Rev. Lett. 110, 126401 (2013).

[26] M. I. Katsnelson and A. I. Lichtenstein, Phys. Rev. B 61, 8906 (2000).

[27] M. I. Katsnelson and A. I. Lichtenstein, Eur. Phys. J. B 30, 9 (2002).

[28] M. Eckstein and P. Werner, Phys. Rev. B 84, 035122 (2011).

[29] R. Sensarma, D. Pekker, E. Altman, E. Demler, N. Strohmaier, D. Greif, R. Jördens, L. Tarruell, H. Moritz, and T. Esslinger, Phys. Rev. B 82, 224302 (2010).

[30] Z. Lenarč and P. Prelovšek, Phys. Rev. Lett. 111, 016401 (2013).

[31] B. Moritz, A. F. Kemper, M. Sentef, T. P. Devereaux, and J. K. Freericks, Phys. Rev. Lett. 111, 077401 (2013).

[32] Y. Nagaoka, Phys. Rev. 147, 392 (1966).

[33] V. Turkowski and J. K. Freericks, Phys. Rev. B 71, 085104 (2005).

[34] J. Nishitani, K. Kozuki, T. Nagashima, and M. Hangyo, Appl. Phys. Lett. 96, 221906 (2010).

[35] M. K. Liu, B. Pardo, J. Zhang, M. M. Qazilbash, S. J. Yun, Z. Fei, J.-H. Shin, H.-T. Kim, D. N. Basov, and R. D. Averitt, Phys. Rev. Lett. 107, 066403 (2011). 